\title{
Detrimental effect of self-phase modulation on the performance of Brillouin distributed fiber sensors
}

\author{
Stella M. Foaleng, ${ }^{1, *}$ Félix Rodríguez-Barrios, ${ }^{2}$ Sonia Martin-Lopez, ${ }^{2}$ \\ Miguel González-Herráez, ${ }^{3}$ and Luc Thévenaz ${ }^{1}$ \\ ${ }^{1}$ EPFL Swiss Federal Institute of Technology, Group for Fibre Optics, Institute of Electrical Engineering, \\ STI IEL GR-SCI-LT Station 11, CH-1015 Lausanne, Switzerland \\ ${ }^{2}$ Instituto de Óptica, CSIC C/ Serrano 144, Madrid 28006, Spain \\ ${ }^{3}$ Departamento de Electrónica, Universidad de Alcalá, Escuela Politécnica DO-231, 28871, Spain \\ *Corresponding author: stella.foalengmafang@epfl.ch
}

Received October 20, 2010; revised December 1, 2010; accepted December 2, 2010; posted December 3, 2010 (Doc. ID 136902); published January 6, 2011

We show that the spectral broadening of the pump pulse through self-phase modulation in a time-domain distributed Brillouin sensor has a considerably detrimental effect in the measurement, especially in the case of long distances and high-resolution pulses. Using $30 \mathrm{~ns}$ pump pulses with peak power of $276 \mathrm{~mW}$, self-phase modulation leads to a doubling of the effective gain linewidth after some $20 \mathrm{~km}$, which is equivalent to a contrast loss of $2 \mathrm{~dB}$ in the measurement. The impact is higher for shorter pulses (higher resolution). The theoretical modeling is fully confirmed by experimental results. (c) 2011 Optical Society of America

OCIS codes: $290.5900,190.2640,060.2370,190.4370,190.3270$.

For at least two decades, Brillouin fiber sensors have attracted great interest in the fiber-sensing community for their temperature and strain-monitoring capability [1,2]. In time-domain-distributed Brillouin sensors, pulses are used to interrogate the local interaction in the fiber. The accuracy on the measurand is scaled by the spectral spreading of the effective gain, which, at its turn, is given by the convolution between the pulse spectrum and the natural Brillouin gain spectrum (BGS). According to standard time-bandwidth relations, the Gaussian pulse is presumably the best candidate for this interrogation when compared to other profiles (rectangular, triangular). However, we show here that this is not the best choice when addressing long ranges, because this pulse shape leads to a significant spectral broadening of the BGS along the fiber. An observed broadening of the BGS was suspected to be caused by self-phase modulation (SPM) in an early work by Lecoeuche et al. [3] and by Izumita et al. [4] in a coherent optical time-domain reflectometer system. SPM leads to small phase chirps during intensity transitions in the pump pulse (leading and trailing edges) that eventually become important in long fibers. The frequency broadening associated with this phase modulation leads to a reduced peak gain and uncertainties in the determination of the Brillouin shift $\nu_{B}$, but it leaves the temporal intensity distribution of the pump pulse unchanged, and hence the spatial resolution is preserved. Although the former [3-5] works showed a correct intuition addressing qualitatively the issue, either no theoretical model was given or the model was incomplete [5]. We present here a quantitative model of the detrimental impact of SPM supported by a clear experimental demonstration. Two optical pulses with different temporal profiles were judiciously chosen (rectangular and Gaussian) showing the same FWHM and carrying the same energy, to evenly study and compare the SPM impact on their spectrum. Then we clearly experimentally demonstrate the spectral broadening of the BGS due to SPM in a Brillouin distributed sensor in various conditions in terms of pump pulse temporal profiles, power, and width. The re- sults are compared with a theoretical model showing good agreement.

SPM is a consequence of the nonlinear Kerr effect in the fiber that results in an intensity-dependent refractive index. It leads an optical pulse to modulate its own optical phase according to its intensity profile. Because the pulse creates a time-dependent refractive index variation, higher for larger intensities, it leads to a time-dependent nonlinear phase shift $\phi_{\mathrm{NL}}$ that translates into variations of the instantaneous frequency (frequency chirp) along the pulse. Let us consider the propagation of an optical pulse through a fiber characterized by a nonlinear refractive index $n(t)=n_{0}+n_{2} I(t)$. After propagation over a distance $z$, the optical pulse accumulates an additional nonlinear phase [6] due to the intensity-dependent part $\phi_{\mathrm{NL}}=n_{2}\left(\omega / c_{0}\right) z \bar{I}(t)$ of the refractive index, which creates a variation in its instantaneous frequency:

$$
\Delta \omega(t)=\frac{d \phi_{\mathrm{NL}}(z, t)}{d t}=-n_{2} \frac{\omega}{c_{0}} z \frac{d I(t)}{d t} .
$$

Equation (1) clearly shows that SPM will have more impact on fast signals constantly showing temporal transitions, with no interval of constant intensity. For our study we have compared two different temporal profiles (rectangular and Gaussian) showing the same width (for equal spatial resolutions) and the same energy (to make gains equal). The rectangular pulse offers constant intensity intervals only, while the Gaussian pulse varies constantly in intensity but shows the best time-bandwidth product (and thus the best time-frequency resolution). Since the intensity is constant at any time for the rectangular profile (assuming instantaneous transitions), $\Delta \omega(t)=0$, and no pulse spectral broadening should therefore be observed. The situation will be drastically different in the Gaussian case showing continuous time transitions. Because it can be entirely described analytically, let us consider a pulse with a Gaussian envelope, $E(0, t)=A \exp$ $\left(-t^{2} / \tau^{2}\right)$, so, $I(t)=\left|A \exp \left(-t^{2} / \tau^{2}\right)\right|^{2}=I_{0} \exp \left(-2 t^{2} / \tau^{2}\right)$ with a $1 / e$ width $\tau$. The instantaneous frequency chirp imposed 
on the Gaussian pulse by SPM using Eq. (1) can be found in Eq. (2) as

$$
\begin{aligned}
\Delta \omega(t) & =4 n_{2} \frac{\omega}{c_{0}} z I_{0} \frac{t^{2}}{\tau^{2}} \exp \left(-2 t^{2} / \tau^{2}\right) \\
& =4 \gamma z P \frac{t}{\tau^{2}} \exp \left(-2 t^{2} / \tau^{2}\right),
\end{aligned}
$$

where $c_{0}$ is the vacuum light velocity; $\omega$ is the central angular frequency of the optical pulse; $I_{0}$ and $P$ are, respectively, the peak intensity and peak power of the optical pulse; $A_{\text {eff }}$ is the effective area of the fiber mode; and $\gamma=$ $n_{2} \omega / c_{0} A_{\text {eff }}$ is the fiber nonlinear coefficient. In a long-fiber segment, the distance $z$ must be replaced by the effective nonlinear distance $z_{\text {eff }}=[1-\exp (-\alpha L)] / \alpha$, where $L$ is the physical length and $\alpha$ is the linear loss of the fiber. The peak excursion of the instantaneous frequency is $\Delta \omega_{\max }=$ $2 \gamma P z_{\text {eff }} / \sqrt{e} \tau=1.43 \gamma P z_{\text {eff }} / T$ offset by $\tau_{\max }=T / \sqrt{8 \ln 2}$ from the pulse center and, as scale factor, gives a good estimate of the real spectral broadening. For typical values in conventional long-range Brillouin optical time-domain analysis (BOTDA) systems $\left(\gamma=1 \mathrm{~W}^{-1} \mathrm{~km}^{-1}, P=100 \mathrm{~mW}\right.$, $z_{\text {eff }}=20 \mathrm{~km}$, and $T=10 \mathrm{~ns}$ ), this quantity amounts to $2 \pi \cdot 29 \mathrm{MHz}$, which is similar to the Brillouin natural linewidth.

A more precise evaluation of the spectral broadening can be obtained by computing the pulse spectrum evolution along the fiber. The actual frequency spectrum of the Gaussian pulse after experiencing SPM in the fiber is given by the Fourier transform of the output pulse amplitude according to Eq. (3):

$$
\begin{aligned}
& g_{\mathrm{SPM}}(\Delta \nu) \\
& =\mathrm{FT}\{\underbrace{A \exp \left(-t^{2} / \tau^{2}\right)}_{\text {Gaussianamplitude term }} \underbrace{\exp \left[i \gamma L_{\text {eff }} P \exp \left(-2 t^{2} / \tau^{2}\right)\right]}_{\text {SPMterm }}\},
\end{aligned}
$$

where, for convenience, the time origin is considered always placed at the pulse center. The effective Brillouin gain linewidth is given by the convolution between the real pump source spectrum after SPM, $g_{\mathrm{SPM}}(\Delta \nu)$, and the natural BGS. It must be noted that all along this derivation we have neglected the effect of chromatic dispersion. This is due to the comparatively long pulse lengths used in BOTDA systems (tens of nanoseconds). It can be easily shown that in these conditions, the dispersion length far exceeds the nonlinear length, and thus dispersion effects can be neglected [6]. In relation to this, modulation instability (which results from the SPM phase matched by anomalous dispersion) is known to impose a limit in the peak power of the pump pulses used in Brillouin sensors owing to nonlinear pump depletion [7]. The observed effect here is radically different from the one observed in [7], because it depends not only on the pulse power but also on the pulse shape and duration, and it causes a smooth broadening of the BGS that was not observed in [7].

The experimental configuration, shown in Fig. 1, is based on a minor adaptation of the high-performance pump and probe setup described in [8]. A $1552 \mathrm{~nm}$ distributed-feedback laser is used as the light source. Pump and signal waves are derived from the same optical source by means of optical modulation and are inserted

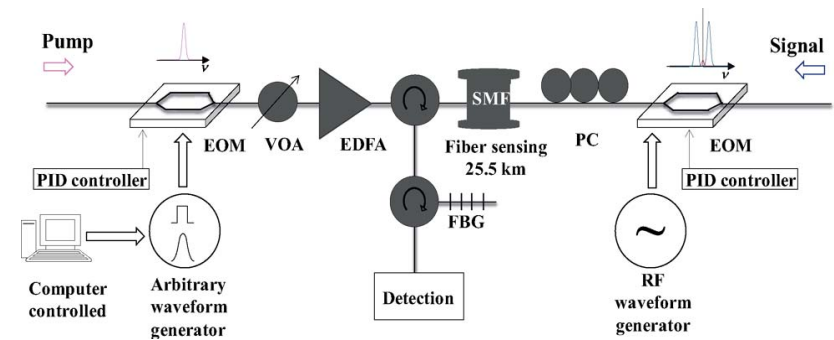

Fig. 1. (Color online) Diagram of the setup: EDFA, erbiumdoped fiber amplifier; EOM, electro-optic modulator; FBG, fiber Bragg grating (0.1 nm spectral width); PC, polarization controller; VOA, variable optical attenuator; and PID, proportionalintegrator circuit.

in the fiber through opposite ends. The pump pulse is shaped by an intensity modulator driven by an arbitrary waveform generator. The produced pulses have $30 \mathrm{~ns}$ FWHM optical pulse duration, corresponding to $3 \mathrm{~m}$ spatial resolution. This duration was carefully chosen for a better demonstration as a trade-off between temporal transitions fast enough to show the SPM effect and long enough to keep the original spectrum sufficiently narrow (to avoid an excessive broadening of the effective BGS). The output light is controlled by a polarization controller to best align the pump and signal polarizations in order to maximize the SBS interaction and then boosted by a highpower EDFA. The BGS is determined by scanning the microwave generator frequency around the Brillouin shift $\nu_{B}$. The net Brillouin gain is then measured using a detection stage comprising a $125 \mathrm{MHz}$ bandwidth photoreceiver and a digital oscilloscope synchronously triggered by the arbitrary waveform generator. The experimental study of the SPM impact on Brillouin sensors has been carried out through a $25.5 \mathrm{~km}$ standard single-mode fiber (SMF) using the above-described BOTDA system. The distributed nature of the measurements makes possible retrieving the effective BGS at any position along the fiber, and thus observing the gradual impact of SPM. Depletion is avoided by using sufficiently low probe power levels (in the microwatt level) to guarantee depletion values below $1 \%$.

Figure 2(a) shows the effective gain linewidth (solid lines) along the fiber using 30 ns FWHM Gaussian pulses for increasing pump peak power from $98 \mathrm{~mW}$ to $276 \mathrm{~mW}$. The graph shows clearly a quasi-linear increase in the effective gain linewidth caused by the pump spectral broadening due to SPM along the fiber. The same figure represents the calculation of the effective gain linewidth (FWHM) as a function of the distance (dotted curves) showing a good match between the experimental result and the theoretical model with $\gamma=0.9 \mathrm{~W}^{-1} \mathrm{~km}^{-1}$. This value of $\gamma$ is a bit small in comparison with the values reported usually in conventional SMF $\left(1.1 \mathrm{~W}^{-1} \mathrm{~km}^{-1}\right)$, but the design of this fiber turns out to be quite outdated (manufactured in 1992), and the relatively low measured Brillouin gain suggests a nonlinear effective area for this fiber larger than usual. Figure 2(b) represents the evolution of the effective gain linewidth along the fiber using 30 ns FWHM rectangular and Gaussian pulses of identical energy (the peak power of rectangular pulse is $222 \mathrm{~mW}$ ). It can be observed that the gain spectral width is doubled after $25.5 \mathrm{~km}$ for the Gaussian pulse, while it 


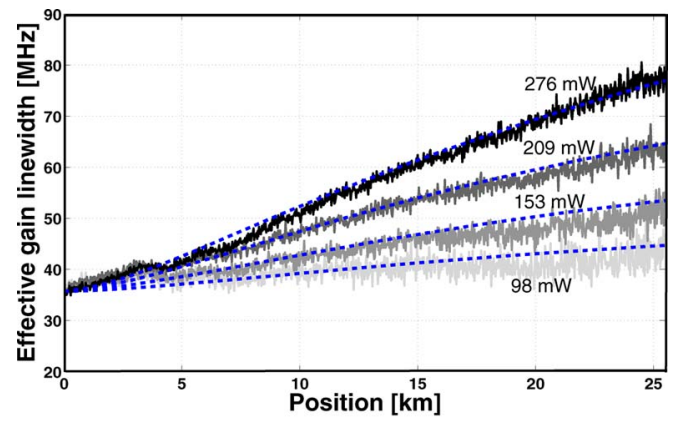

(a)

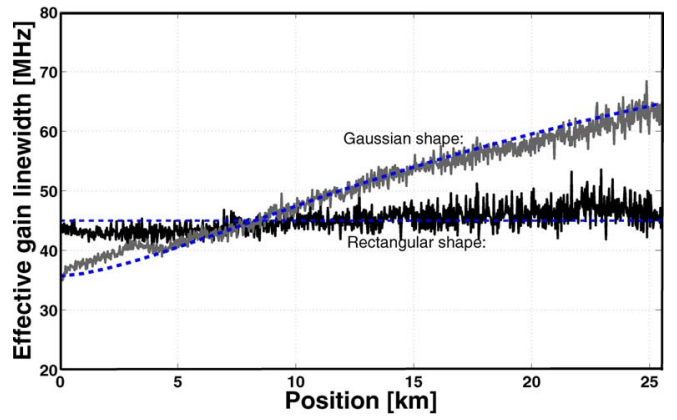

(b)

Fig. 2. (Color online) Experimental demonstration (solid lines) and theoretical analysis (dotted curves) of the gain spectrum broadening due to SPM along a $25.5 \mathrm{~km}$ SMF (a) for different peak powers of a 30 ns FWHM Gaussian pulse and (b) comparing 30 ns FWHM Gaussian and rectangular pulses of identical energy (peak power of rectangular pulse is $222 \mathrm{~mW}$ ).

remains nearly unchanged for the rectangular pulse as predicted by the model (dotted curves). The residual broadening of the spectrum in the square pulse is probably due to a nonideal shape in the rectangular pulse used (the rise and fall edges are not ideally sharp). This incidentally confirms a negligible pump depletion that would instead broaden the effective linewidth of the gain spectrum near the fiber end, independently of the pulse shape. The benefit of a better time-bandwidth product using the Gaussian pulse turns out to be entirely canceled by SPM after less than $10 \mathrm{~km}$. From Fig. 2(a), and considering the extreme case of $276 \mathrm{~mW}$ peak power, the contrast loss can be estimated at the output of the fiber $(25.5 \mathrm{~km})$ to be $2 \mathrm{~dB}$, which is equivalent to a $10 \mathrm{~km}$ distance penalty in standard conditions.

Figure 3(a) represents comparative measurements of the gain spectral width measured for a Gaussian pulse at the fiber input (dark gray diamonds) and output (light gray squares), respectively, for different peak powers, demonstrating the clear signature of SPM by the absence of effect at a short distance and through the linear dependence on power at long distances. As shown in Fig. 3(b), the effective Brillouin gain linewidth varies in inverse proportion to the optical pulse duration $T$ at both the fiber input and output, but with a different slope. An excess broadening is present at the output as a result of the pump spectral spreading. This excess broadening becomes larger for shorter pulses, showing also a $1 / T$ slope, in good agreement with the theory above.

In conclusion, we have demonstrated that SPM-induced spectral broadening can have a significant effect on the

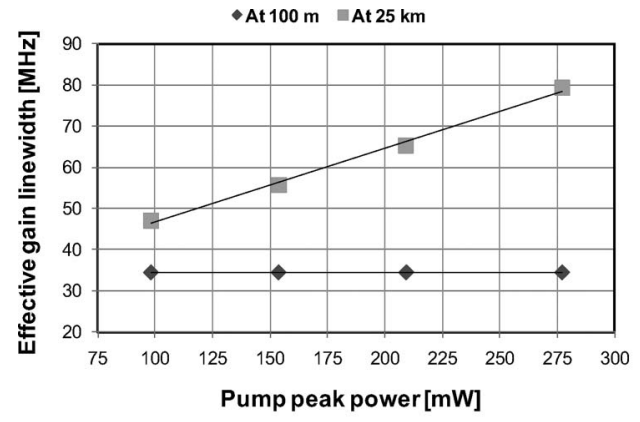

(a)

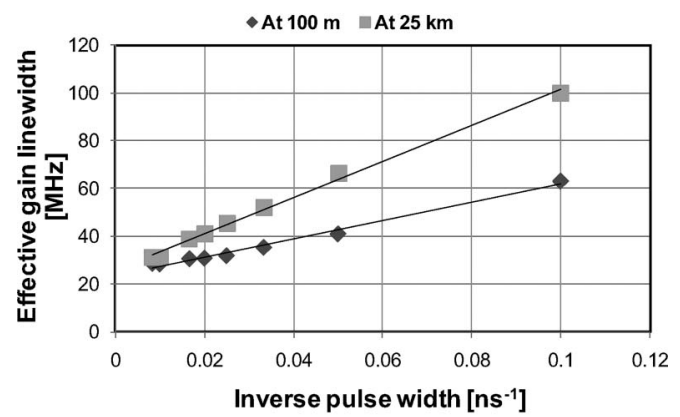

(b)

Fig. 3. Brillouin gain spectral width measured close to the fiber input $(100 \mathrm{~m})$ and at the fiber output $(25.5 \mathrm{~km})$ using a 30 ns Gaussian pulse: (a) linear dependence on the pump peak power, showing that the effect of SPM is observed only at the distant end and (b) inverse dependence of the gain linewidth on the pulse width, showing the excess broadening due to SPM at the far end (pump power is $153 \mathrm{~mW}$ ).

effective gain linewidth measured by Brillouin sensors. Modeling and experiments have undoubtedly demonstrated that the effective gain linewidth can easily experience a twofold increase in standard conditions when the pulse intensity profile is Gaussian. At power and spatial resolutions used in standard configurations, spectral broadening can be observed typically from a $5 \mathrm{~km}$ distance. Practically, the problem can be circumvented by using a clean rectangular pulse with very sharp rising and falling edges. The benefit of a narrower spectrum for an identical pulse width brought by the Gaussian pulse is rapidly canceled after about $10 \mathrm{~km}$ for $209 \mathrm{~mW}$ pump peak power.

\section{References}

1. T. Kurashima, T. Horuguchi, and M. Tateda, Opt. Lett. 15, 1038 (1990).

2. M. Nikles, L. Thévenaz, and P. A. Robert, Opt. Lett. 21, 758 (1996).

3. V. Lecoeuche, D. J. Webb, C. N. Pannell, and D. A. Jackson, Opt. Commun. 168, 95 (1999).

4. H. Izumita, Y. Koyamada, S. Furukawa, and I. Sankawa, J. Lightwave Technol. 12, 1230 (1994).

5. S. M. Foaleng, F. Rodríguez-Barrios, S. Martin-Lopez, and M. González-Herráez, and L. Thévenaz, Proc. SPIE 7653, 76532U (2010).

6. R. W. Boyd, Nonlinear Optics, 4th ed. (Academic, 2008).

7. M. N. Alahbabi, Y. T. Cho, T. P. Newson, P. C. Wait, and A. H. Hartog, J. Opt. Soc. Am. B 21, 1156 (2004).

8. S. Diaz, S. M. Foaleng, M. Lopez-Amo, and L. Thévenaz, IEEE Sensors J. 8, 1268 (2008). 